\title{
PROPERTIES OF CERTAIN SPACES OF ENTIRE FUNCTIONS
}

\section{MINAKETAN DAS}

1. A. C. Offord [3] has discussed the behaviour of Fréchet's space $\mathcal{F}$ of entire functions. He has shown that "most" (viz. those belonging to a set whose complement is of the first category in F) entire functions behave in a rather wild manner.

In this note we show that "most" entire functions of $\mathcal{F}$

(i) are of lower order zero,

(ii) possess certain properties which are ordinarily met with the polynomials "only" (cf. (1), (2) and (3) below).

We mention here some of the properties of the space $\mathcal{F}$, viz:

(a) A sequence of entire functions is convergent if it converges uniformly on every closed bounded set of the complex plane.

(b) $\mathfrak{F}$ is a complete metric space.

(c) The set of all polynomials is dense in $\mathscr{F}$.

It follows from (b) that $\mathcal{F}$ is of the second category. The properties (a), (b) and (c) happens to be possessed by the space $\Gamma$ of all entire functions, considered by V. Ganapathy Iyer in [2].

Our methods are variants of those of Offord, who like us, uses only the properties (a)-(c) mentioned above. As such all his results and those below hold for Iyer's space too.

We denote the number of zeros of a function $f(z)$ in the open region $|z|<r$ by $n_{*}(r, f)$; also $N$ denotes a fixed positive integer. We prove

THEOREM. Let $r_{1}, r_{2}, r_{3}, \cdots$ be an increasing and unbounded set of positive constants. Then there is a set $\mathcal{F}^{*}$ of entire functions whose complement is of the first category in $\mathfrak{F}$. Let $f \in \mathcal{F}^{*}$. Then.

(1) $n\left(r_{k}, f\right)-n_{*}\left(r_{k}, f^{(N)}\right) \leqq N,\left(f^{(N)} \equiv d^{N} f / d z^{n}\right)$,

(2-a) $\left(\log r_{k}\right) n_{*}\left(r_{k}, f\right) \geqq \log M\left(r_{k}, f\right)$,

(2-b) $\left(\log r_{k}\right) n\left(r_{k}, f\right) \leqq \log M\left(r_{k}, f\right)$, and

(3) $M\left(r_{k}, f^{(N)}\right) r_{k}^{N}<M\left(r_{k}, f\right) \geqq 1$

for an infinity of $k$ in each case. The sets of $k$ 's depend on $f$ and are not necessarily the same in the above.

(4) Each $f$ of $\mathfrak{F}^{*}$ has lower order zero.

We shall first prove a

Lemma. If $\left\{f_{\nu}(z)\right\}$ is a sequence of entire functions which converge to $f(z)$ on every bounded closed set, then there is an integer $\nu^{\prime}=\nu(r)(d e-$ pending on $r$ ) such that

Received by the editors April 4, 1967. 


$$
n_{*}(r, f) \leqq n_{*}\left(r, f_{v}\right) \leqq n\left(r, f_{v}\right) \leqq n(r, f) \text { for all } \nu>\nu^{\prime} .
$$

Proof of the Lemma. Let $\zeta_{1}, \zeta_{2}, \cdots, \zeta_{k}$ denote the distinct zeros of $f$ in $|z|<r$ and $\zeta_{k+1}, \cdots, \zeta_{k+k^{\prime}}$ those on $|z|=r$. By a well-known theorem of Hurwitz, these points together and these only are the limit points of the zeros of the sequence $\left\{f_{\nu}\right\}$. We enclose each $\zeta_{s}$ by sufficiently small neighbourhoods $N_{s}$ and choose

$$
\nu_{s} \quad\left(s=1,2, \cdots, k+k^{\prime}\right)
$$

such that

(a) the $N_{s}$ are nonoverlapping,

(b) $N_{1}, \cdots, N_{k}$ lie in $|z|<r$ and

(c) if $\zeta_{s}$ is an $m$-fold zero of $f(z), N_{s}$ contains exactly $m$ zeros (counted according to their multiplicities) of each $f_{\nu}$ for $\nu>\nu_{s}$.

Only the zeros of a finite number of $\left\{f_{\nu}\right\}$, say with $\nu<\nu_{0}$, lie in the region which is the complement of $N_{1} \cup \ldots \cup N_{k+k^{\prime}}$ in $|z| \leqq r$. Further an infinity of $f_{\nu}$ may vanish in those parts of $N_{k+1}, N_{k+2}, \cdots$, $N_{k+k^{\prime}}$ which lie on either side of $|z|=r$. The lemma follows on taking

$$
\nu(r)=\operatorname{Max}\left\{\nu_{0}, \nu_{1}, \cdots, \nu_{k+k^{\prime}}\right\} .
$$

2. We take up the relation (1) to be proved. For this, let $\left\{f_{v}(z)\right\}$ be any sequence of entire functions which converge to an arbitrarily given entire function $f(z)$ on each of the closed sets $|z|=r_{n}$ $(n=1,2, \cdots)$. By the maximum modulus principle and the elementary property of inclusion of bounded sets in a system of concentric circles, it follows that $\left\{f_{\nu}\right\}$ converges to $f$ "in $F$." Let $E_{p}$ be the sets of entire functions for which

$$
n\left(r_{p}, F\right)-n_{*}\left(r_{p}, F^{(N)}\right) \geqq N+\text { i }
$$

holds. By our lemma, $E_{p}$ is closed and hence,

$$
\mathcal{E}_{p}=\underset{\nu \geq p}{\bigcup} E_{\nu}
$$

is also closed. Let

$$
\varepsilon=\underset{p}{U} \varepsilon_{p} \quad(p=1,2, \cdots) .
$$

If

$$
f(z)=\sum_{k=0}^{\infty} a_{k} z^{k}
$$

be any arbitrarily given entire function, let 


$$
g_{m}(z)=\sum_{k=0}^{m} a_{k} z^{k}
$$

Clearly $g_{m}(z)$ belongs to the complement of $E_{p}$ for all sufficiently large $p$ since the relation

$$
n\left(r, g_{m}\right)-n_{*}\left(r, g_{m}\right) \leqq N
$$

holds for all sufficiently large $r$. Thus $g_{m}$ belongs to the complement of $\varepsilon_{p}$ in $\mathcal{F}$ for every $p$. But $f$ is any entire function and hence the complement of $\varepsilon_{p}$ is everywhere dense in $\mathcal{F}$. Thus $\varepsilon_{p}$ is a frontier set. But every $\varepsilon_{p}$ is closed. Therefore each $\mathcal{E}_{p}$ is nondense. $\varepsilon$ is, therefore, the union of a denumerable infinity of nondense sets; that is, $\mathcal{E}$ is of the first category.

Suppose $\phi$ satisfies (2.1) for $p \geqq k$. Then $\phi \in E_{k^{\prime}}$, for $k^{\prime} \geqq k$, i.e.

$$
\phi \in \mathcal{E}_{k} \subset \mathcal{E} \text {. }
$$

Conversely, if $\phi \in \mathcal{E}$, then $\phi \in \mathcal{E}_{k}$ for some $K$, i.e. $\phi \in E_{k^{\prime}}$ for $k^{\prime} \geqq k$. Hence $\phi$ satisfies (2.1) for all large $p$. Thus

$$
\mathcal{E}=\left\{f ; n\left(r_{p}, f\right)-n_{*}\left(r_{p}, f^{(N)}\right)>N \text { for } p>p_{0}(f)\right\}
$$

is of the first category. So are each of the following sets.

$$
\begin{aligned}
& \mathcal{G}_{k}=\left\{f ;\left(\log r_{p}\right) n_{*}\left(r_{p}, f\right) / \log M\left(r_{p}, f\right)<1-1 / k, \text { for } p>p_{1}(k, f)\right\}, \\
& \mathfrak{H}_{k}=\left\{f ;\left(\log r_{p}\right) n\left(r_{p}, f\right) / \log M\left(r_{p}, f\right)>1+1 / k, \text { for } p>p_{2}(k, f)\right\} \text {, } \\
& \mathfrak{g}=\left\{f ; M\left(r_{p}, f^{(N)}\right) r_{p}^{N} / M\left(r_{p}, f\right)<1, \text { for } p>p_{3}(f)\right\}, \\
& g_{k}=\left\{f ; \log \log M\left(r_{p}, f\right) / \log r_{p}>1 / k, \text { for } p>p_{4}(k, f)\right\}, \\
& \mathcal{G}=\mathcal{G}_{1} \cup \mathcal{G}_{2} \cup \mathcal{G}_{3} \cup \ldots \text {, } \\
& \mathfrak{H}=\mathfrak{H}_{1} \cup \mathfrak{H}_{2} \cup \mathfrak{H}_{3} \cup \ldots \text { and } \\
& \mathcal{d}=\mathfrak{d}_{1} \cup \mathfrak{d}_{2} \cup \mathfrak{d}_{3} \cup \ldots .
\end{aligned}
$$

If $\psi$ does not belong to $\varepsilon$, then $\psi$ lies outside $\varepsilon_{p}$ for every $p$, i.e. $\psi$ does not belong to $E_{\nu}$ for an infinity of $\nu$. Hence $\psi$ violates (2.1) for an infinity of $p$. Let,

$$
\mathfrak{F}^{*}=\mathfrak{F}-(\mathcal{E} \cup \mathcal{G} \cup \mathfrak{H} \cup \mathfrak{g} \cup \mathcal{g}) .
$$

By the above, for any $\psi$ belonging to $\mathfrak{F}^{*},(2.1)$ is violated for an infinity of $p$. This proves part (1) of our theorem. The relations (2-a), $(2-b)$ and (3) are similarly verified for any $\psi \in \mathcal{F}^{*}$. Now $\mathcal{g}$ is precisely the set of those entire functions whose lower orders are positive, and the proof of our theorem is complete. 


\section{REFERENCES}

1. T. D. Baum, Elements of point set topology, Prentice-Hall, Englewood Cliffs, N. J., 1964; p. 139.

2. V. Ganapathy Iyer, On the space of integral functions, J. Indian Math. Soc. 12 (1948), 13-30.

3. A. C. Offord, Some remarks on Frechet's space of integral functions, Proc. London Math. Soc. (3) 2 (1952), 62-69.

Fakir Mohan College, Balasore, India 